\title{
Infectious keratitis with corneal perforation associated with corneal hydrops and contact lens wear in keratoconus
}

Eric D Donnenfeld, Amilia Schrier, Henry D Perry, Herbert J Ingraham, Richard Lasonde, Arthur Epstein, Bruce Farber

\begin{abstract}
Background-Corneal perforation is an uncommon complication associated with keratoconus. The first cases of infectious keratitis and corneal perforation associated with corneal hydrops and contact lens wear are reported in two keratoconus patients.

Methods-A retrospective chart review and histopathological examination were carried out.

Results-Both patients progressed to corneal perforation and emergency penetrating keratoplasty. One patient cultured Fusarium and the second patient Serratia marcesens. Both patients wore contact lenses against medical advice.

Conclusions-The tear in Descemet's membrane, stromal oedema, and epithelial bedewing associated with corneal hydrops results in loss of the epithelialendothelial barrier of the cornea, creating a conduit for infectious organisms through the cornea. Acute hydrops associated with epithelial keratitis, stromal swelling, and a Descemet's membrane tear may be a significant risk factor for infectious keratitis and corneal perforation. Contact lenses should not be worn during an active corneal hydrops owing to the increased risk for severe infectious keratitis and corneal perforation.

(Brf Ophthalmol 1996; 80: 409-412)
\end{abstract}

Department of Ophthalmology, North Shore University Hospital, Manhasset, New York and Cornell University Medical College, New York, USA

E D Donnenfeld

A Schrier

H D Perry

$R$ Lasonde

A Epstein

B Farber

Department of

Ophthalmology,

Geisinger Medical

Center, Danville,

Pennsylvania, USA

$\mathrm{H} \mathrm{J}$ Ingraham

Correspondence to:

Eric Donnenfeld, $M D$, Lions

Eye Bank for Long Island at

North Shore University

Hospital, Manhasset, NY

11030, USA.

Accepted for publication

19 January 1996
Infectious keratitis associated with contact lens wear is a well described entity. ${ }^{1-15}$ Risk factors for contact lens associated microbial keratitis include poor hygiene, tear film abnormalities, extended wear contact lens use, and epithelial defects. Severe keratitis is generally associated with Gram negative infection ${ }^{245-10}$ and/or a compromised host. Corneal perforation is an extremely rare complication of contact lens related microbial keratitis. A large series of contact lens associated corneal ulcers ${ }^{5}$ found one corneal perforation in 54 consecutive patients in which the contact lens was worn for visual rehabilitation.

Corneal hydrops, a rupture of Descemet's membrane with secondary stromal and epithelial oedema, is a dramatic occurrence in keratoconus. ${ }^{16}$ The symptoms are related to the acute break in Descemet's membrane with stromal and epithelial oedema. Visual acuity is acutely compromised and patients may complain of pain and photophobia. Resolution of the epithelial and stromal oedema generally occurs over a period of months, often with concomitant improvement in visual acuity. We have recently managed two keratoconus patients with infectious keratitis who progressed to corneal perforation despite aggressive antimicrobial therapy. Both patients gave a history of keratoconus with contact lens wear in the presence of an acute corneal hydrops.

\section{Methods}

A thorough retrospective chart review was undertaken on both patients. In addition, a histopathological examination of corneal specimens was performed.

\section{Case reports}

CASE 1

A 51-year-old woman presented with a 1 day history of acute pain and decreased vision in the right eye. Previous ocular history was pertinent for keratoconus, managed with a gas permeable contact lens in the right eye. Six weeks before presentation, the patient had developed acute corneal hydrops in the right eye with epithelial and stromal oedema. She was managed with sodium chloride $5 \%$ drops four times daily, prednisolone acetate $1 \%$ four times daily, and chloramphenicol $0.5 \%$ drops twice daily. The steroids were tapered and discontinued after 10 days; the patient was advised not to wear contact lenses. Four weeks before presentation, the patient travelled to the

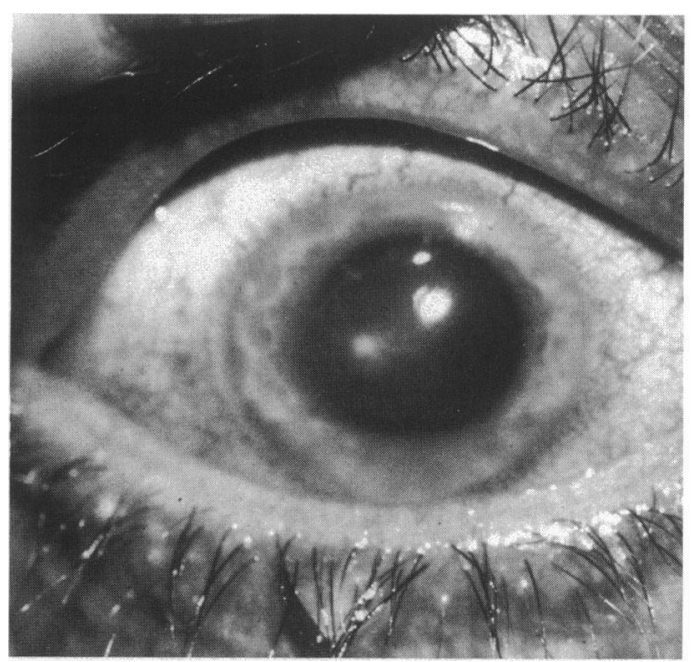

Figure 1 Case 1, day 1. Photograph showing deep stromal corneal infiltrate. 


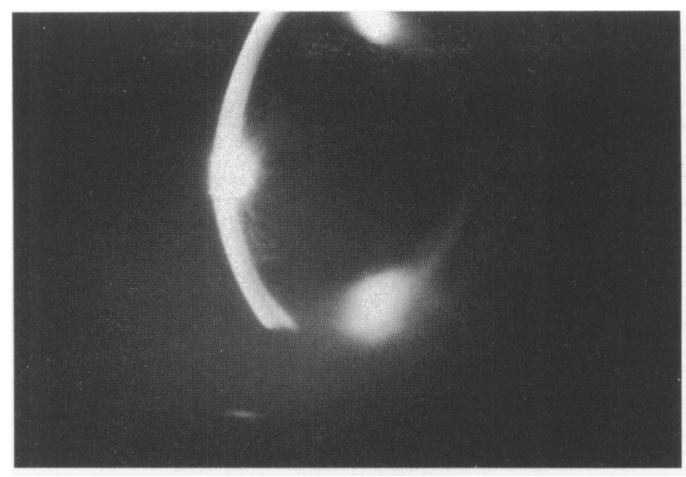

Figure 2 Case 1, day 1. Slit-lamp photograph showing normal anterior corneal stroma with inflammatory cells radiating from the endothelium into the anterior chamber.

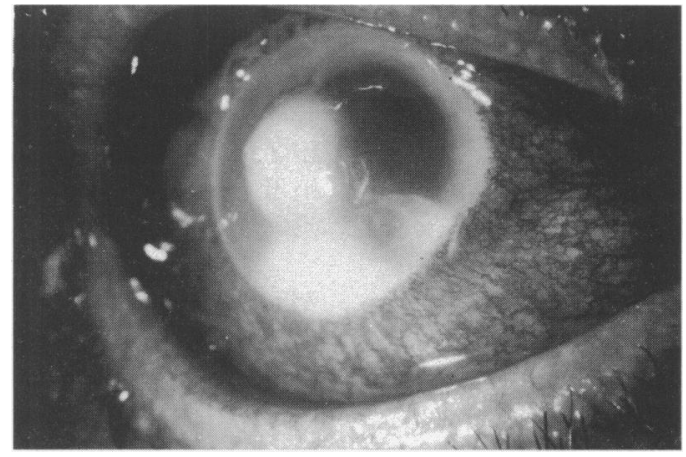

Figure 3 Case 1, day 7. Photograph showing central corneal infiltrate with central perforation and $15 \%$ inferior hypopyon.

Caribbean, wore the contact lenses, experienced pain, and treated herself with prednisolone acetate $1 \%$ every 2 hours and chloramphenicol $0.5 \%$ drops four times daily. Following this, the patient wore contact lenses again, experienced acute pain, and once again, treated herself with chloramphenicol $0.5 \%$ and prednisolone acetate $1 \%$ drops in the right eye.

On presentation, ocular examination revealed a visual acuity of $20 / 200$ in the right eye and 20/20 in the left eye. The conjunctiva was $3+$ diffusely injected in the right eye. The epithelium was clear and intact. There was a $1.5 \mathrm{~mm}$ endothelial plaque immediately beneath the tear in Descemet's membrane with inflammatory cells radiating into the anterior chamber (Figs 1 and 2). The overlying stroma was compact and not inflamed. The intraocular pressure was $16 \mathrm{~mm} \mathrm{Hg}$ in both eyes. The

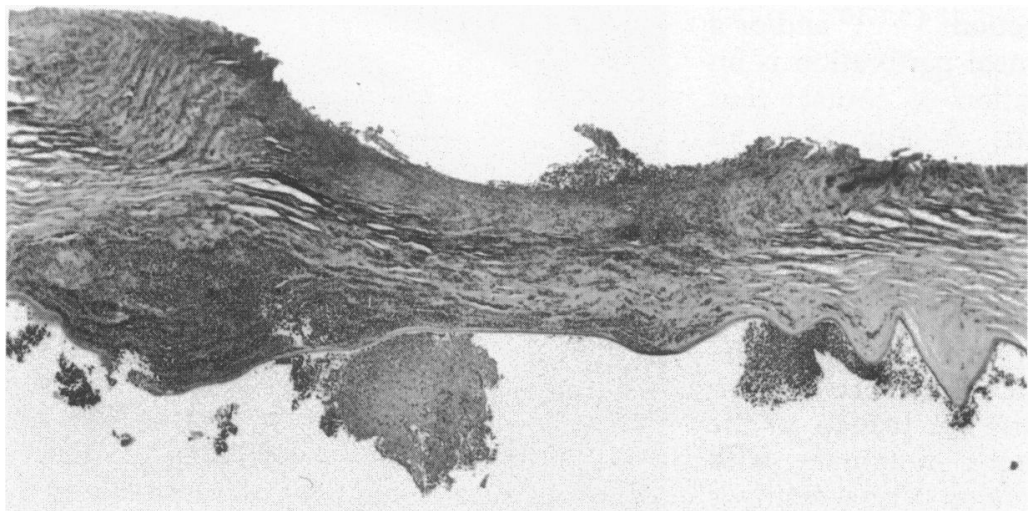

Figure 4 Case 1. Photomicrograph $(\times 36)$. Corneal pathology revealing severe corneal thinning with inflammatory stromal infiltrates and adherent hypopyon. lens was clear. The vitreous was clear. The posterior pole was normal.

The patient underwent anterior chamber paracentesis with removal of the inflammatory plaque. Gram, Giemsa, and silver stains were negative. Topical treatment was initiated consisting of chloramphenicol $0.5 \%$ drops four times daily and amphotericin $0 \cdot 1 \%$ drops every 2 hours. After 24 hours, Fusarium was identified on three culture media, later speciated to Fusarium solani. The patient was started on amphotericin $0.4 \%$ drops every 15 minutes, natamycin 5\% drops every hour, fluconazole $100 \mathrm{mg}$ orally twice daily, and doxycycline $100 \mathrm{mg}$ orally twice daily. The patient experienced increasing hypopyon formation, progressive corneal thinning (Fig 3), and went on to corneal perforation on day 10 of treatment.

An emergency $8 \mathrm{~mm}$ penetrating keratoplasty was performed. The hypopyon was cultured. Postoperatively the patient was maintained on natamycin 5\% drops four times daily and fluconazole $100 \mathrm{mg}$ once a day, which was tapered over a 2 month period. Prednisolone acetate $1 \%$ drops were started on postoperative day 5. Intraocular and corneal cultures were negative. Histology of the cornea revealed an adherent hypopyon characteristic of fungal keratitis (Fig 4). Silver stain demonstrated the presence of organisms throughout the corneal stroma, despite 10 days of antifungal therapy. Six months postoperatively, following suture removal, the patient had an uncorrected vision of $20 / 25$ in the right eye.

\section{CASE 2}

A 36-year-old man was referred for evaluation with complaints of decreased vision and pain in the left eye of 1 day duration. Ocular history was significant for keratoconus in both eyes, managed with rigid gas permeable contact lenses for 6 years. There was a history of acute hydrops with epithelial bedewing in the left eye for 1 month, which was treated by the referring physician with tobramycin $0.3 \%$ /dexamethasone $0.1 \%$ ophthalmic solution four times daily. During this time, the patient continued to wear contact lenses against medical advice.

On ocular examination, the visual acuity was $20 / 80$ in the right eye with contact lens correction, and hand motion in the left eye. The conjunctiva in the left eye was $3+$ injected with a marked limbal flush. The corneal examination revealed a $7 \mathrm{~mm}$ inferior paracentral corneal ulcer with $5 \mathrm{~mm}$ of central corneal thinning. The surrounding cornea was noted to have a marked stromal infiltrate and a significant purulent discharge (Fig 5).

Pneumotonometry of the periphery was 16 . The patient was Seidel negative. There was a $5 \%$ inferior hypopyon and a pupillary membrane was noted. B-scan ultrasound was normal. Diagnostic corneal scrapings for culture and sensitivity, as well as Gram, Giemsa, and Papanicolaou stains were obtained.

The patient was empirically started on cefazolin drops, $100 \mathrm{mg} / \mathrm{ml}$, and tobramycin drops, $14 \mathrm{mg} / \mathrm{ml}$, alternating every half hour. The patient was given one subconjunctival injection 


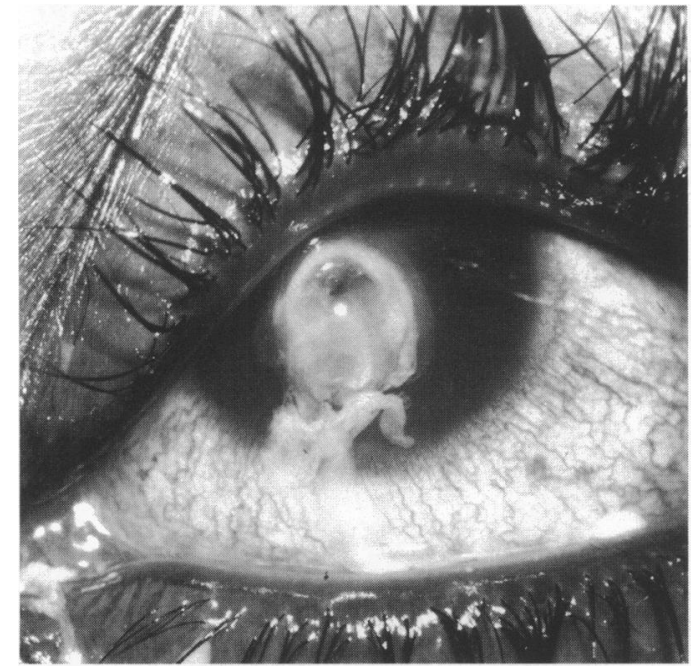

Figure 5 Case 2. Cornea had marked stromal infiltrate and a significant purulent discharge.

of tobramycin $80 \mathrm{mg}$, and cefazolin $200 \mathrm{mg}$. Serratia marcesens was cultured from the cornea within 48 hours. Antibiotic therapy was modified to tobramycin $14 \mathrm{mg} / \mathrm{ml}$ drops every hour. The hypopyon resolved, but the patient had progressive corneal thinning. Despite 10 days of aggressive therapy, the patient went on to a $4 \mathrm{~mm}$ corneal perforation.

An emergency $7.5 \mathrm{~mm}$ penetrating keratoplasty was performed. The patient was maintained on chloramphenicol $0.5 \%$ and dexamethasone phosphate $0 \cdot 1 \%$ ophthalmic solutions postoperatively. Pathology of the cornea adjacent to the area of thinning revealed a marked inflammatory infiltrate and stromal extracellular fluid with a loss of normal collagen lamellae (Fig 6). Six months postoperatively, with sutures in place, the patient corrected to $20 / 30$ visual acuity.

\section{Results}

Both patients progressed to corneal perforation despite aggressive antimicrobial therapy. Both patients also required emergency penetrating keratoplasty. One patient cultured Fusarium and the second patient Serratia marcesens. A histopathological examination in both eyes revealed significant corneal thinning, consistent

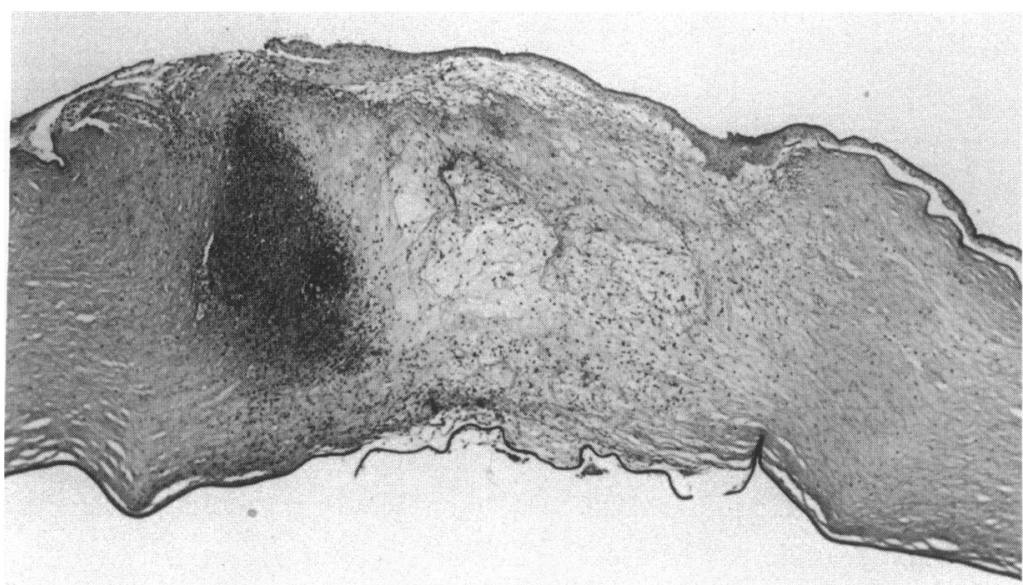

Figure 6 Case 2. Photomicrograph $(\times 36)$ of keratoplasty specimen adjacent to the area of perforation, revealing inflammatory cellular infiltrate in the corneal stroma contiguous to the area of extracellular stromal oedema. with the diagnosis of keratoconus. In addition, there were marked inflammatory infiltrates and loss of normal collagen lamellae. In the case associated with Fusarium viable fungal organisms were visible on pathology and cultured, despite 10 days of aggressive antifungal therapy. Both patients did well with penetrating keratoplasty and achieved good visual acuity with a mean follow up of 11 months.

\section{Discussion}

These two cases demonstrate contact lens wear, epithelial defects, and corticosteroids as risk factors for infectious keratitis. Contact lens wear in the presence of an epithelial defect permits the adherence of micro-organisms to the lens, prolonging the contact time of pathogens to the corneal surface. ${ }^{13}$ Corneal swelling associated with contact lens wear alone may cause enough epithelial disruption to precipitate corneal infection. ${ }^{12}{ }^{13}$ In the two cases presented, there was epithelial bedewing secondary to corneal hydrops and contact lens wear, allowing an entry site for the infectious organisms.

Spontaneous corneal perforation associated with corneal hydrops in the absence of infection has recently been described by Ingraham, et al. ${ }^{17}$ Following penetrating keratoplasty for visual rehabilitation, pathology of the involved cornea revealed a prominent fistula. The fistula tracked directly from the tear in Descemet's membrane through the entire stroma to Bowman's membrane. This fistula could easily act as a conduit, enabling organisms to track into the deep stroma and anterior chamber. As the infection progressed, the fistula could certainly enlarge sufficiently to become an overt perforation.

The normal corneal stroma is composed of collagen fibres. The collagen fibres are arranged in lamellar sheets, which lie at oblique angles to one another. Each lamella is continuous across the cornea. In stromal oedema, the interlamellar spaces are widened, and irregularity of the collagen lamellae occurs. ${ }^{18}$ We believe that the stromal oedema associated with corneal hydrops produces a change in the normal architecture of the corneal lamellae, facilitating the passage of organisms through the cornea. In keratoconus, there is also a decrease in the number of stromal lamellae associated with the corneal thinning. ${ }^{16}$ Decreased number of collagen lamellae with concomitant disruption may be responsible for the rapid penetration of infectious organisms through the keratoconic cornea with acute hydrops.

These two cases demonstrate stromal oedema and tears in Descemet's membrane to be significant risk factors for corneal perforation in infectious keratitis. In both patients, the ulcerative keratitis and perforation occurred directly over the area of hydrops. The acute tear in Descemet's membrane in hydrops allows not only access of fluid from the anterior chamber into the corneal stroma, but also causes a loss of the barrier function of 
Descemet's membrane and endothelium. The resultant stromal oedema produces a change in the normal architecture of collagen lamellae, weakening the integrity of the cornea. Infectious keratitis and corneal perforation associated with bandage contact lens wear have been reported in patients with bullous keratopathy. ${ }^{519}$ Spontaneous corneal perforation is a rare, but well documented occurrence in corneal hydrops. ${ }^{17}$ The combination of corneal oedema and a rupture of Descemet's membrane creates a conduit, allowing infectious organisms to pass readily from the surface of the eye into the anterior chamber.

In conclusion, we feel contact lens wear in the face of acute hydrops increases the risk of severe infectious keratitis and corneal perforation.

This study was supported in part by the Lions Club International Foundation, Oakbrook, Illinois.

1 Schein OD, Ormerod LD, Barraquer E, Alfonso E, Egan KM, Paton BG, et al Microbiology of contact lens-related keratitis. Cornea 1989; 8: 281-5.

2 Ormerod LD, Smith RE. Contact lens-associated microbial keratitis. Arch Ophthalmol 1988; 104: 79-83.

3 Schein OD, Poggio EC. Ulcerative keratitis in contact lens wearers. Cornea 1990; 9 (suppl 1): S55-8.

4 Galentine PG, Cohen EJ, Laibson PR, Adams CP, Michaud $\mathrm{R}$, Arentsen JJ. Corneal ulcers associated with contact lens wear. Arch Ophthalmol 1984; 102: 891-4.
5 Donnenfeld E, Cohen E, Arentsen J, Genvert G, Laibson P. Changing trends in contact lens associated corneal ulcers: An overview of 116 cases. CLAO f 1986; 12: 145-9.

6 Stern GA. Pseudomonas keratitis and contact lens wear: the lens/eye is at fault. Cornea 1990; 9 (suppl 1): S36-8.

7 Dart JKG. Predisposing factors in microbial keratitis: the significance of contact lens wear. $\mathrm{Br} \mathcal{F}$ Ophthalmol 1988; 72: $926-30$.

8 Chalupa E, Swarbrick HA, Holden BA, Sjostrand J. Severe corneal infections associated with contact lens wear. Ophthalmology 1987; 94: 17-22.

9 Koidou-Tsiligianni A, Alfonso E, Forster RK. Ulcerative keratitis associated with contact lens wear. $A m$ Ophthalmol 1989; 108: 64-7.

10 Alfonso E, Mandelbaum S, Fox MJ, Forster RK. Ulcerative keratitis associated with contact lens wear. $A m f$ Ophthalmol 1986; 101: 429-33.

11 Mondino BJ, Weissman BA, Farb MD, Pettit TH. Corneal ulcers associated with daily wear and extended-wear con-

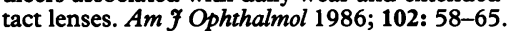

12 Koch JM, Refojo MF, Hanninen LA, Leong FL, Kenyon KR. Experimental Pseudomonas aeruginosa keratitis from extended wear of soft contact lenses. Arch Ophthalmol 1990; 108: 1453-9.

13 Stern GA. Corneal infection in contact lens wearers. In Ophthalmol Clinics, Spring 1991; 31: 147-61.

14 Wilhelmus KR, Robinson NM, Font RA, Hammel MB Jones DB. Fungal keratitis in contact lens wearers. Am Jones DB. Fungal keratitis in cont

15 Wilson LA, Ahearn DG. Association of fungi with extended-wear soft contact lenses. Am $\mathcal{F}$ Ophthalmol 1986; 101: 434-6.

16 Krachmer JH, Feder RS, Belin MW. Keratoconus and related non-inflammatory corneal thinning disorders. Surv Ophthalmol 1984; 28: 293-322.

17 Ingraham HJ, Donnenfeld ED, Perry HD. Keratoconus with spontaneous perforation of the cornea. Arch with spontaneous perforation

18 Douthman DJ. Corneal edema. Clinical ophthalmology. Vol 4. Philadelphia: Harper \& Row, 1985: 1-17.

19 Andrew NC, Woodward EJ. A bandage lens in bullous keratopathy. Ophthal Physiol Optics 1988; 9: 66-8. 